\title{
Heavy Fermions and Superconductivity: Ten Years After
}

\author{
F. Steglich, Darmstadt \\ (Institut für Festkörperphysik, Technische Hochschule, D-6100 Darmstadt)
}

\begin{abstract}
The 1989 Hewlett-Packard Europhysics Prize was awarded jointly to Professor Frank Steglich, Professor Hans R. Ott of ETH Zurich and Dr. Gilbert Lonzarich of the Cavendish Laboratory, Cambridge, for their pioneering investigations of heavy fermion metals. In addition to the intrinsic interest of these unforeseen materials they are ideal for testing models of other physical phenomena such as band magnetism and superconductivity.
\end{abstract}

In 1979, superconductivity was discovered below $T_{\mathrm{c}} \cong 0.6 \mathrm{~K}$ for the compound $\mathrm{CeCu}_{2} \mathrm{Si}_{2}$ [1]. Since the trivalent $\mathrm{Ce}$ ions in this material carry local moments associated with the singly occupied $4 \mathrm{f}$-shells, a magnetically ordered rather than a superconducting ground state was to be expected. However, upon cooling $\mathrm{CeCu}_{2} \mathrm{Si}_{2}$ to below a characteristic temperature, $T^{*} \cong 15 \mathrm{~K}$, the local moments become quenched, and the system approaches a metallic ("Fermi-liquid") low-temperature phase. These observations are reminiscent and, in fact, closely related [2] to the so-called "Kondo effect" as frequently observed in dilute magnetic alloys like $\mathrm{CuFe}$ or LaCe.

The finding of bulk superconductivity in a trivalent cerium intermetallic was surprising for most experimentalists, as usually, less than 1 at $\% \mathrm{Ce}^{3+}$ dopant suffices to suppress superconductivity in a BCS superconductor: an exchange interaction between the conductionelectron spins and the spins of the localized $4 \mathrm{f}$-shells at the $\mathrm{Ce}$ sites breaks the Cooper pairs. The pair-breaking capability of paramagnetic ions demonstrates the antagonistic relationship between magnetism and classical superconductivity: sufficiently localized (3d-, $5 f$ - and $4 \mathrm{f}-$ ) electrons, whose motion is dominated by their intra-atomic Coulomb correlations, give rise to magnetic phenomena, whereas conduction-electron systems of more delocalized (s-, p-, $5 d$-, $4 d-$-) electrons favour a superconducting state.

$\mathrm{CeCu}_{2} \mathrm{Si}_{2}$ was the first example exhibiting "superconductivity of strongly correlated electrons", in that its 4 f electrons are responsible not only for the local magnetic moments but also for superconductivity. This can be inferred from Fig. 1a, which shows that the specific heat discontinuity at $T_{c}$ is of the same gigantic size as the normalstate specific heat, $C_{n}\left(T_{c}\right)$, which is of electronic origin since the phonon contribution can be completely neglected at these low temperatures. The asymptotic specific heat behaviour in the normal state as extrapolated from above $T$ to $T \rightarrow O$ is $C_{n}=\gamma T$, where the coefficient $\gamma\left(\cong 1 \mathrm{~J} / \mathrm{K}^{2} \mathrm{~mol}\right)$ exceeds that of simple metals like $\mathrm{Al}$ or $\mathrm{Pb}$ by two to three orders of magnitude. $\gamma$ indicates the effective masses of the low-energy excitations ("quasiparticles") of the normal Fermi-liquid phase which are approximately 300 times larger than the free-electron mass, $m_{\mathrm{e}}$. These "heavy fermions" can be interpreted as $4 \mathrm{f}$ electrons that have been weakly delocalized by a Kondo-type effect and, thus, have become itinerant states right at the Fermi energy, $E_{\mathrm{F}}[2]$. Both the extremely

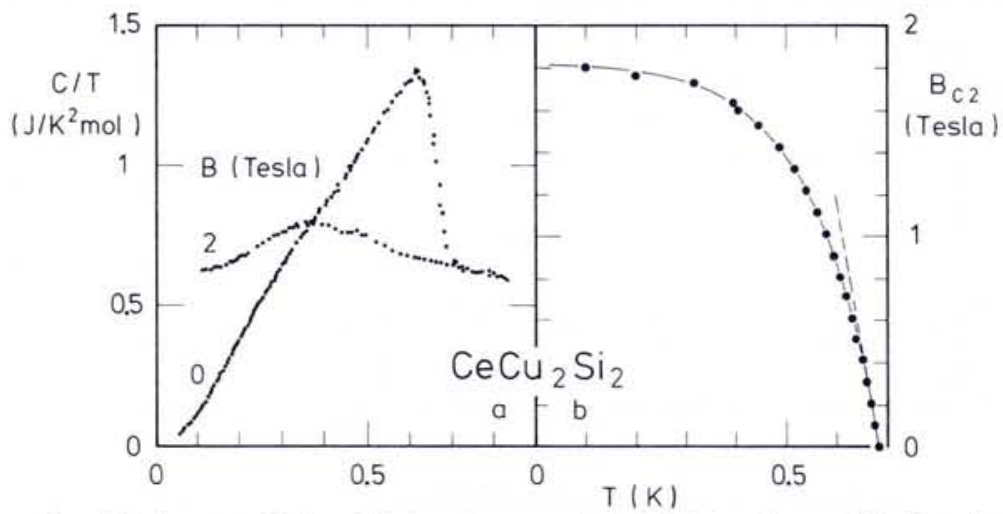

Fig. 1 - Specific heat as $C / T$ vs $T$ (a) and upper critical field as $B_{\mathrm{c} 2} v s T$ (b) for the same sample of $\mathrm{CeCu}_{2} \mathrm{Si}_{2}$ (Steglich F. et al., Physica 126B (1984) 82). Dashed line in (b) indicates the giant $B_{c 2}(T)$ slope at $T_{c}(-13 T / K)$. large specific heat jump height and the very steep slope of the curve of the upper critical magnetic field $B_{c 2}(T)$, separating the superconducting and the normal phases (Fig. 1b), prove that the Cooper pairs in $\mathrm{CeCu}_{2} \mathrm{Si}_{2}$ are formed by the heavy fermions. Consequently, the homologue $\mathrm{LaCu}_{2} \mathrm{Si}_{2}$ with no $4 \mathrm{f}$ electrons and no heavy-fermion properties is not a superconductor.

\section{Kondo Lattice}

Although for all heavy-fermion compounds the separation of neighbouring f-ions is much too large to allow a significant $f$-wavefunction overlap, hybridization with ligand orbitals tends to destabilize the magnetic configuration of the $\mathrm{f}$-shell. In the case of weak hybridization, the Kondo effect is relevant [2], while stronger hybridization may give rise to instabilities of the f-occupation, i.e. via charge or "valence" fluctuations [4]. For most heavy-fermion compounds, however, inter-site couplings compete with the single-site Kondo interaction and cause a magnetically ordered state to develop in the range of liquid helium temperatures. The archetypical systems $\mathrm{CeAl}_{2}$ and $\mathrm{NpSn}_{3}$, both studied in the 1970s, are characterized by reduced ordered magnetic moments (compared with those of the corresponding free f-ions) and strongly enhanced electronic specific heats (compared with their non-f homologues, e.g., $\mathrm{LaAl}_{2}$ and $\mathrm{LaSn}_{3}$ ), see Figs. 2a and $\mathrm{b}$ : the Kondo coupling sets in already at elevated temperatures and gradually reduces the local moments upon cooling. This process is associated with a gradual piling up of a resonance at the Fermi energy, indicating an increasing effective mass of the quasiparticles [2]. For an illustration, the increase of the coefficient $\gamma$ (being proportional to $m^{*}$ ) is shown in Fig. 2a, as it would be expected within the Kondo-impurity model for fictitious paramagnetic $\mathrm{CeAl}_{2}$. In reality, the magnetic interactions dominate 


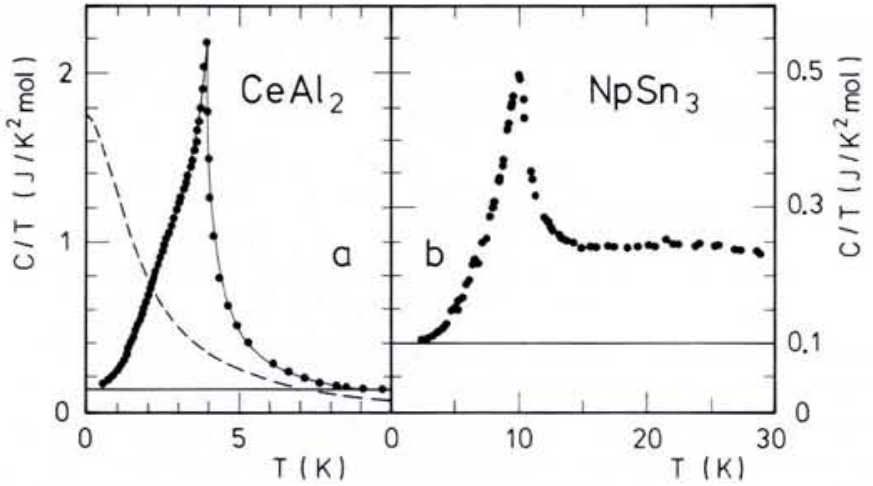

Fig. $2-C / T$ vs $T$ for $\mathrm{CeAl}_{2}$ (Bredl C.D. et al., J. Magn. Magn. Mat. 9 (1978) 60) (a) and $\mathrm{NpSn}_{3}$ (Trainor R.J. et al., Phys. Rev. Lett. 34 (1976) 1019) (b). Solid lines mark specific heat coefficient $\gamma$ (as $T \rightarrow 0$ ). Dashed curve in (a) represents result of $S=1 / 2$ Kondo-impurity model (the Kondo temperature $T_{k}$ is chosen to equal the characteristic temperature $\left.T^{*}=3.5 \mathrm{~K}\right)$.

over the Kondo interaction at low $T$, so that long-range antiferromagnetism develops below the Néel temperature $T_{N}=3.9 \mathrm{~K}$.

For a relatively small number of compounds, this type of ordering between local moments does not occur since the demagnetizing interaction finally wins. Even in the absence of long-range magnetic order, however, heavy-fermion compounds behave qualitatively differently from their related dilute alloys. This is demonstrated in Fig. 3a which gives the resistivity results by Yoshio Onuki and Takemi Komatsubara for the quasi-binary system $\mathrm{La}_{1-x} \mathrm{Ce}_{x} \mathrm{Cu}_{6}$. At low $\mathrm{Ce}$ concentration, the $\mathrm{Ce}^{\prime} \mathrm{s}$ are distributed at random on the La sites and are subject to a Kondo effect. The electrical resistivity which approaches its maximum as $T$ approaches absolute zero and decreases continuously upon warming, demonstrates the action of a resonance scattering: heavy fermions become formed locally, as can also be inferred from the anomalous rise in $\gamma(T)$ displayed in Fig. 2a. For the compound $\mathrm{CeCu}_{6}$ a qualitatively similar resistivity curve is found well above the characteristic temperature $T^{*} \cong 4 \mathrm{~K}$, whereas a quite different behaviour is observed at low temperatures. Most importantly, the resistivity reaches a maximum near $T^{*}$ rather than as $T \rightarrow 0$. This demonstrates that, as a consequence of the translational symmetry in the Ce subsystem, the incoherent resonance scattering disappears. No matter how anomalous the scattering potential of the single $\mathrm{Ce}$ ions, upon building a periodic lattice, at sufficiently low temperature they give rise to "coherent scattering": the scattering processes between the heavy fermions, represented by extended ("Bloch") states well below $T=$ $T^{*}$, give rise to a contribution $A T^{2}$ to the resistivity, which is a hallmark of a (charged) Fermi liquid. Since the coeffiabout the low- $T$ Fermi-liquid state of a Kondo lattice is derived from its instabiPhys. Rev. Lett. 35 (1975) 1779). lity against superconducting and magnetic phase transitions.

\section{Cooperative Phenomena}

In contrast to experimentalists, theorists seemed to look with favour on the observation of heavy-fermion superconductivity in $\mathrm{CeCu}_{2} \mathrm{Si}_{2}$ : the ratio $T_{\mathrm{c}} / T_{\mathrm{F}^{\prime}}^{*}$ where $k_{B} T_{F}^{*}$ is the effective width of the heavy-fermion band at $E_{\mathrm{F}}$, greatly exceeds the ratio $T_{c} / T_{F}$ for classical superconductors, whose conduction-band width is of the order of the Fermi energy $E_{\mathrm{F}}=k_{\mathrm{B}} T_{\mathrm{F}}$. It is even considerably larger than $T_{\mathrm{c}} / T_{\mathrm{F}}$ for the cuprate superconductors. Therefore, heavy-fermion compounds may be considered "high- $T_{\text {c }}$ superconductors" in a figurative sense. In particular, an estimate of the renormalized Fermi velocity reveals the same order of magnitude as for the velocity of sound. As a result, the phase space for the BCS-type coupling mechanism appears to be strongly reduced, so that the latter came under early suspicion.

By analogy to the Fermi liquid ${ }^{3} \mathrm{He}$, which turns superfluid in the mK-range, a non-phononic Cooper-pairing mechanism was presupposed. In ${ }^{3} \mathrm{He}$, shortlived magnetic excitations ("'spin fluctuations") instead of phonons are assumed to be essential for the formation of Cooper pairs. In addition, the on-site Coulomb repulsion between heavy fermions, which resembles the hard-core repulsion between two ${ }^{3} \mathrm{He}$ atoms, is invoked in arguments against an s-wave Cooper-pair state as in a BCS superconductor. A node of the pair-wave function (or the order parameter) is expected to occur at the origin of the relative coordinate [2]. So continuing the analogy to ${ }^{3} \mathrm{He}$, whose Cooper pairs are in a spin-triplet state, while BCS superconductors exhibit singlet pairs, $\mathrm{CeCu}_{2} \mathrm{Si}_{2}$ was assumed to be the first triplet superconductor.

However, this possibility was quickly discarded by the subsequent investigations: while the large initial slope of

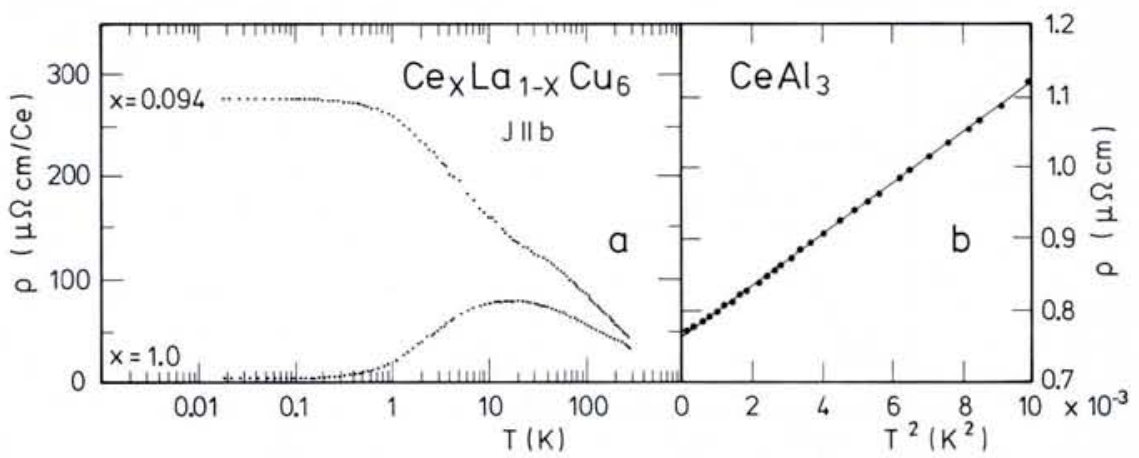

Fig. 3 - a: Ce increment of the electrical resistivity for the dilute Kondo alloy La $\mathrm{L}_{0.906}$ $\mathrm{Ce}_{0.094} \mathrm{Cu}_{6}$ and the "Kondo lattice" $\mathrm{CeCu}_{6}$ (Ōnuki Y. and Komatsubara T., J. Magn. Magn. Mat. 63 \& 64 (1987) 281). b: Resistivity vs $T^{2}$ for $\mathrm{CeAl}_{3}$ below $T=0.1 \mathrm{~K}$ (Andres K. et al., 
$B_{c 2}(T)$ results from a "diamagnetic effect", which acts on the momentum pairing of the Cooper pairs via the Lorentz force, the pronounced flattening of the $B_{\mathrm{c} 2}(T)$ curve at lower temperatures (Fig. $1 \mathrm{~b}$ ) proves the importance of a second (paramagnetic) pair-breaking effect of the external magnetic field, which acts on the spin state of the Cooper pairs. In a rotational-invariant system like ${ }^{3} \mathrm{He}$ this so-called "Pauli limiting" should be absent for triplet pairs. Measurements of the orientation dependence of the Pauli-limit for $\mathrm{CeCu}_{2} \mathrm{Si}_{2}$ single crystals revealed that triplet pairing in this material is most unlikely. Additional support for this conclusion was derived from careful measurements of the DC-Josephson current through weak links between $\mathrm{CeCu}_{2} \mathrm{Si}_{2}$ and clear-cut BCS superconductors like $\mathrm{Al}$ and $\mathrm{Nb}$. Whereas in the case of triplet-superconducting $\mathrm{CeCu}_{2} \mathrm{Si}_{2}$ no Josephson effect would be expected prior to ordering, a Josephson current of the size typical for singlet-singlet junctions was observed.

Though presumably a singlet superconductor, $\mathrm{CeCu}_{2} \mathrm{Si}_{2}$ as well as the three $\mathrm{U}$-based heavy-fermion superconductors $\mathrm{UBe}_{13}, \mathrm{UPt}_{3}$ and $\mathrm{URu}_{2} \mathrm{Si}_{2}$ (as discovered in 1983 and 1984 by Hans Ott, Greg Stewart, Wolfram Schlabitz and their collaborators) seem to exhibit highly anisotropic order parameters. This is inferred, for example, from the non-exponential temperature dependence of the low-temperature specific heat (see Fig. 4a) and related transport properties. Small values of the superconducting energy gap in certain directions lead to an increased density of excited states at low energies which raises the low- $T$ specific heat above the (exponential) BCS result. No agreement can presently be achieved, however, as to the type of order-parameter anisotropy. Arguments have been presented in favour of both "unconventional" and "conventional" order parameters whose symmetry is either lower than or equal to the symmetry of the Fermi surface. Since the de-Haas - van-Alphen measurements as well as theoretical considerations [3] reveal strong Fermisurface anisotropies, conventional order parameters in HFS would also have to be highly anisotropic.

Among unconventional order parameters, two different general classes are distinguished according to grouptheoretical considerations: the first class involves zeros of the energy gap along lines on the Fermi surface and is represented by, e.g., a (singlet) d-wave function. It should be manifested well

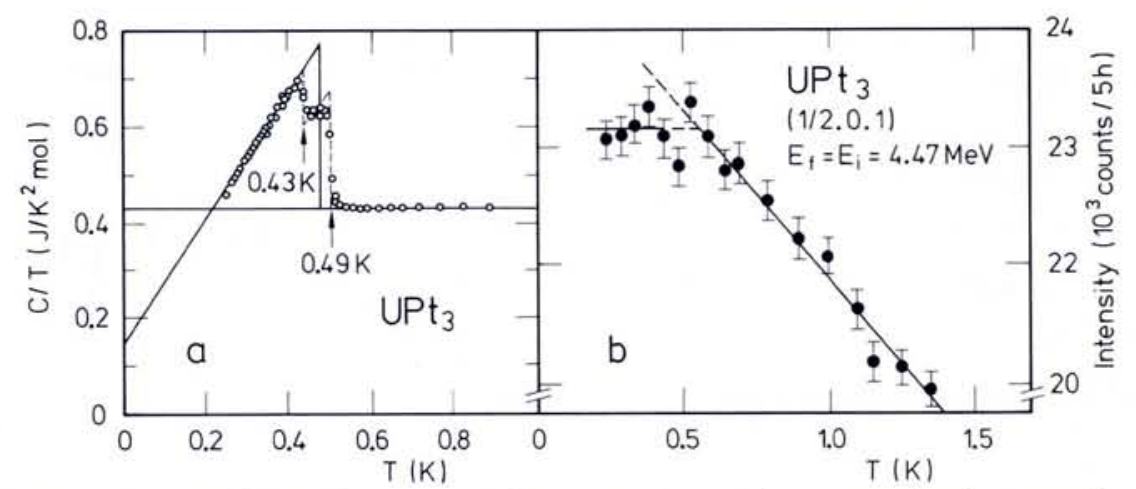

Fig. $4-U_{P_{2}}$ : (a) $C / T$ vs $T$ results reveal two closely spaced superconducting transitions at $0.49 \mathrm{~K}$ and $0.43 \mathrm{~K}$. If they are replaced by a single transition, this would be located at $0.47 \mathrm{~K}$. Extrapolation of the superconducting data to $T-0$ suggests a dependence $C_{\mathrm{s}}=$ $\gamma_{\mathrm{s}} T+\alpha_{\mathrm{s}} T^{2}$ (Fisher R.A. et al., Phys. Rev. Lett. 62 (1989) 1411). (b) Neutron-scatteringintensity results for the $(1 / 2,0,1)$ reflex as a function of temperature indicate interference between superconductivity and antiferromagnetic order (Aeppli G. et al., Phys. Rev. Lett. 60 (1988) 615).

below $T_{c}$ by a $T^{2}$ dependence of the specific heat. According to Fig. 4a, $\mathrm{UPt}_{3}$ may be a d-wave superconductor. The second class of unconventional order parameters is characterized by gap zeros at certain symmetry points on the Fermi surface as expected with, e.g., (triplet) p-wave superconductors. Such materials should show a $T^{3}$ behaviour of the specific heat as $T \rightarrow 0$ in apparent agreement with experimental results for $\mathrm{UBe}_{13}$. A triplet state of $\mathrm{UBe}_{13}$ has also been inferred from the $T^{2}$-dependence of the magnetic pene-

tration depth shown in Fig. 5a. On the other hand, a $T^{3}$-dependence of the nuclear spin-lattice relaxation rate was found for this material and interpreted as evidence for d-wave superconductivity. This illustrates that the observation of "simple power laws" in heavyfermion superconductors may be fortuitous. Sometimes a thorough analysis of experimental data yields no power laws at all, whilst they are often registered close to $T_{\text {c }}$ (cf. Fig. 4a) when they ought to occur far below $T_{c}$ [3]. In the presence of lattice defects, more

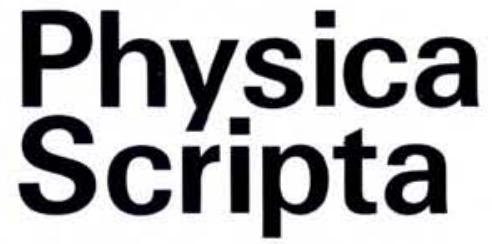

An international journal for experimental and theoretical physics

Vol. T29 1989

\section{Proceedings of the 9th General Conference of the EPS Condensed Matter Division}

Nice, 6-9 March 1989

Edited by: J. Friedel, J.P. Laheurte, J.P. Romagnan

In addition to the texts of six plenary talks the issue contains 54 papers submitted to the Conference. These are arranged in the order in which they were presented.

Although the section on superconductors is particularly significant, of equal importance are the contributions on charge density waves, surfaces and interfaces, semiconductors (e.g. MBE structures and optical phenomena), industrial applications and materials science, phonons and fractons, liquids, polymers and quantum fluids.

A volume of $\mathbf{3 1 0}$ pages of topical information.

Orders to Physica Scripta at the address shown below;

Price : SKR 460.- or US\$70.-

Published by the

ROYAL SWEDISH ACADEMY OF SCIENCES 


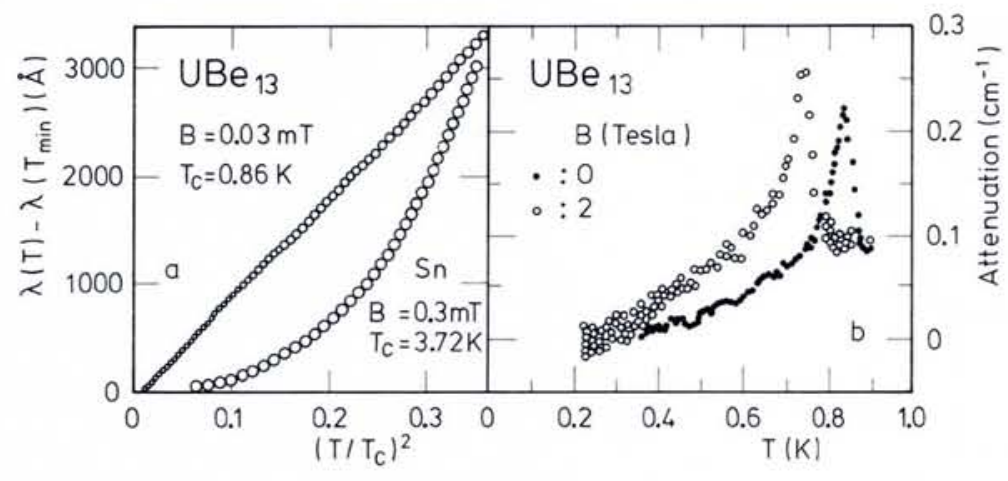

Fig. $5-U_{B e_{13}}$ : (a) increment of the magnetic field penetration depth as a function of $\left(T / T_{c}\right)^{2}$. For comparison, data for the BCS superconductor $\mathrm{Sn}$ are also shown (Einzel D. et al., Phys. Rev. Lett. 56 (1986) 2513). (b) Longitudinal ultrasonic attenuation data taken as a function of temperature at $1.7 \mathrm{GHz}$ for $B=0$ and $2 T$ display the superconducting phase transition (Golding B. et al., Phys. Rev. Lett. 55 (1985) 2479).

complex $T$-dependencies are predicted theoretically [4]. Simple power-law dependencies have been established also for classical superconductors. For example, the specific heat of $\mathrm{HfV}_{2}\left(T_{\mathrm{c}}\right.$ $=9 \mathrm{~K}$ ) is well described by a $T^{3}$ dependence over a large temperature regime.

Anisotropies of the order parameter should be smeared out by potential scattering. In fact, non-magnetic dopants like Th, La and $Y$ partially substituted for the f-ions in $\mathrm{CeCu}_{2} \mathrm{Si}_{2}$ and $\mathrm{UBe}_{13}$ act qualitatively in a way similar to magnetic dopants in a BCS superconductor. On the other hand, the "pair breaking" by non-magnetic scatterers is surprisingly weak if estimated on the assumption of an isotropic potential scattering. This difficulty adds to another serious problem: for both $\mathrm{CeCu}_{2} \mathrm{Si}_{2}$ and $\mathrm{UBe}_{13}$, the mean free path of the heavy fermions estimated from the measured normal-state resistivities are considerably shorter than the superconducting coherence lengths. These observations highlight a strong anisotropy of the scattering rate in Kondo lattices [3] which has to be unravelled by future work.

As with the shape of the superconducting order parameter, the nature of

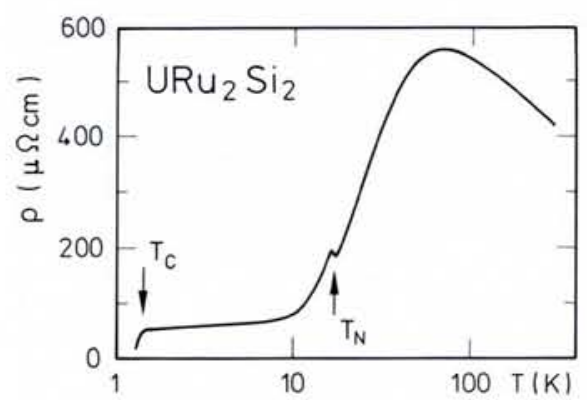

Fig. $6-\mathrm{URu}_{2} \mathrm{Si}_{2}: \rho$ vs $T$ (on a logarithmic scale). Arrows mark the onsets of antiferromagnetism $\left(T_{N}\right)$ and superconductivity $\left(T_{\mathrm{C}}\right)$. Both phenomena are coexisting at low temperatures (Schlabitz W. et al., Z. Phys. B62 (1986) 171). the pairing interaction is presently not known. Arguments in favour of magnetic coupling, in addition to the seeming analogy to superfluid ${ }^{3} \mathrm{He}$, were provided by the Los Alamos group who noticed that superconductivity in $\mathrm{UPt}_{3}$ "possibly coexists" with the pronounced spin-fluctuation phenomena that had been previously discovered for this compound by Jaap Franse and his colleagues. In the meantime, it has been found using sensitive microscopic probes like muon-spin rotation, nuclear magnetic resonance and neutron-diffraction measurements that magnetic correlations dominate the low- $T$ "Fermi-liquid" properties of certain heavyfermion compounds like $\mathrm{CeCu}_{6}$ and $\mathrm{CeRu}_{2} \mathrm{Si}_{2}$ and even give rise to a cooperative antiferromagnetic state in others like $\cup R u_{2} \mathrm{Si}_{2}, \mathrm{UPt}_{3}$ and $\mathrm{U}_{1-\mathrm{x}} \mathrm{Th}_{\mathrm{x}} \mathrm{Be}_{13}$ $(0.01<x<0.05)$. Since for these systems the ordered moments are usually extremely small ( $\lesssim 10^{-2} \mu_{B} / f$-ion), a magnetic instability of the coherent heavyfermion band seems to lead to "band magnetism"' in the Fermi liquid; this effect should be distinguished from the "local-moment ordering" in heavy-fermion systems like $\mathrm{CeAl}_{2}$. The coexistence between this form of band antiferromagnetism and superconductivity is illustrated in Figs. $4 \mathrm{~b}$ and 6 for both $\mathrm{UPt}_{3}$ and $\mathrm{URu}_{2} \mathrm{Si}_{2}$. More than one superconducting phase transition seem to occur in $\mathrm{UPt}_{3}$ (Fig. 4a) and in $\mathrm{U}_{1-\mathrm{x}} \mathrm{Th}_{\mathrm{x}} \mathrm{Be}_{13}$ and they have been associated with both unconventional and conventional order parameters.

Alternatively, an electron-phonon coupling unique for Kondo lattices had been proposed to explain superconductivity with $T_{\mathrm{c}} \leqq 1 \mathrm{~K}$ in $\mathrm{CeCu}_{2} \mathrm{Si}_{2}[2,3]$. It is based upon a pronounced volume dependence of $k_{\mathrm{B}} T^{*}$, the characteristic energy or heavy-fermion band width and can be expressed by a giant "Grüneisen parameter", $\Gamma=-d \ell \mathrm{n}\left(k_{\mathrm{B}} T^{*}\right) /$ $\mathrm{d} \ell \mathrm{nV} \cong 100$. Therefore, the "breathing mode" of the lattice, which is associated with large volume changes, couples strongly to the heavy fermions.

Experimental evidence for this particular electron-phonon coupling is found in striking anomalies of the elastic constants (as determined in Bruno Lüthi's group), thermal expansion and longitudinal sound absorption (see Fig. 5b).

\section{Outlook}

Heavy-fermion systems have provided a number of exciting phenomena which were quite unexpected within traditional concepts of metal physics. They allow us to investigate, for example, how band magnetism develops in a material characterized at elevated temperatures by a dense array of local moments. In addition, they are ideal testing grounds for models concerned with superconductivity in strongly correlated electron systems and they may have some impact on the future understanding of the phenomenon of high- $T_{c}$ superconductivity in $\mathrm{Cu}$-oxides.

\section{REFERENCES}

[1] For an early review, see: Stewart G.R., Rev. Mod. Phys. 56 (1984) 755.

[2] See, e.g.: Fulde P., Europhys. News 16 (1985) 14

[3] For recent reviews, see: Lee P.A., Rice T.M., Serene J.W., Sham L.J. and Wilkins J.W., Comments on Condensed Matter Phys. 12 (1986) 99; Ott H.R., Progr. Low Temp. Phys. XI (1987) 217; Fulde P., Keller J. and Zwicknagl G., Solid State Phys. 41 (1988) 1; Grewe N. and Steglich F., Handbook on the Physics and Chemistry of Rare Earths, Vol. 14, Eds. K.A. Gschneidner Jr. and L. Eyring, forthcoming.

[4] See, e.g.: Müller-Hartmann E., Europhys. News 13 (1982) 7, 9.

[5] Proceedings of the 6th Int. Conf. on Crystal-Field Effects and Heavy-Fermion Physics, Eds. W. Assmus, P. Fulde, B. Lüthi and F. Steglich, Frankfurt (1988), published in J. Magn. Magn. Mat. 76 \& 77 (1988).

\section{Hewlett-Packard Europhysics Prize}

To celebrate the 50th anniversary of the company's foundation and the 30th anniversary of its establishment in Europe, Hewlett-Packard Europe decided to double the value of the $1989 \mathrm{Hew}$ lett-Packard Europhysics Prize from the previous 20000 SFR to 40000 SFR. We are delighted to announce that the company proposes to take this as a precedent and will maintain the figure in the coming years.

The winner(s) of the 1990 HewlettPackard Europhysics Prize will be announced in the February issue of Europhysics News. 